\title{
SHORT-TERM RESULTS OF HIP ENDOPROSTHESIS WITH HEMIPROSTHESIS AFTER FEMORAL NECK FRACTURE MAINTAINING JOINT LUBRICATION IN ADULT PATIENTS
}

\author{
Nikolay Kolarov, Ruslan Popstefanov \\ Orthopedic and Traumatology Clinic, St. Anna University Hospital of Varna, \\ Medical University of Varna
}

\begin{abstract}
INTRODUCTION: Femoral neck fracture is one of the most common traumas in adult patients. Usually, the patients with such fractures present with many accompanying diseases, i. e. polymorbidity. The majority of intraarticular femoral neck fractures are indicated for primary hip joint alloplastic surgery. Despite the higher operational risk compared to osteosynthesis, this procedure is preferred for the treatment of femoral neck fractures in patients over 75 years of age.

The main aim of this study is to build-up optimal indications or guidelines for primary endoprosthesis with hemiprosthesis after femoral neck fractures and to justify the choice of an optimal hip hemiprosthetic implant.

MATERIALS AND METHODS: An one-year study assessed the therapeutic effectiveness of the elliptical monoarticular endoprosthetic head use compared to monoarticular spherical and biarticular spherical endoprosthetic head applications based on three groups of criteria: subjective, radiographic, and functional in 167 patients with hemiarthroplasty.

RESULTS: According to the criteria mentioned above, better therapeutic outcomes have been achieved with the use of the elliptical monoarticular endoprosthetic head. The results of the follow-up of these patients showed that up to the end of the first year after surgery, a progressive elevation in the Modified Harris Hip Score point system as well as a regression to the disappearance of spontaneous and provoked pain regardless of the endoprosthetic design was recorded. The visual analogue scale scores showed a natural decrease in pain sensation with the passing of time from the surgical intervention despite the increasing motor activity.

CONCLUSION: The primary hip joint hemialoplasty is indicated for femoral neck fractures, Garden III or IV, in polymorbid patients over 75 years of age. The elliptical monoarticular endoprosthetic head shows better therapeutic results based on subjective, radiographic and functional criteria during the 12-month follow-up study. Scr Sci Med. 2017;49(4):29-34

Keywords: femoral neck fracture, elliptical hemiprosthetic head, biarticular spherical hemiprosthetic head, monoarticular spherical hemiprosthetic head

Address for correspondence:

Nikolay Kolarov, MD

Clinic of Orthopedics and Traumatology

St. Anna University Hospital

100 Tzar Osvoboditel Blvd

Varna 9002

e-mail:kolarov_ortop@abv.bg

Received: September 1, 2017

Accepted: September 4, 2017

\section{INTRODUCTION}

Femoral neck fracture in adults accounts for $15 \%-25 \%$ of all the hospitalized patients with locomotor injuries. This fracture occurs predominantly in patients over 75 years of age and accounts for $90 \%$ of all the femoral fractures and $65 \%$ of all the bone fractures. The economic costs of treating this fracture are almost $70 \%$ of the overall costs for treating all kinds of fractures.
\end{abstract}


Nowadays, the femoral neck fracture remains a serious therapeutic problem. Proper and timely treatment leads to $70 \%$ patient's survival after fracture, while $40 \%$ of these patients remain with permanent low quality of life due to polymorbidity and later on need additional periodic or permanent social assistance. Besides $24 \%$ of the patients die in the first year after surgery because of fracture-related reasons. Moreover, polymorbidity requires additional preoperative preparation and continuation of the concomitant therapies for the chronic diseases of every patient.

The fracture itself demonstrates specific pathoanatomic features. As a result of the deficient perfusion of the central fracture fragment due to the disruption of the arteries feeding the femoral head during the trauma, the outcome of the fracture treatment is difficult to predict.

The professional guidelines state that the surgical treatment of femoral neck fractures has no alternatives. The purpose of surgical treatment is to restore limb function. Osteosynthesis and the anticipated subsequent bone adhesion results in restoration of the anatomical bone integrity. In comparison, hip joint reconstruction through primary hemiprosthesis allows for an early loading of the operated limb and rapid functional recovery (1).

There is no universal implant for osteosynthesis of the femoral neck. There are mainly two reasons: the complex bone architecture and the usually poor bone quality as a result of osteoporosis. In order to achieve the desired therapeutic goal, postoperative treatment is also required including a specific rehabilitation regimen with the aim to lower the burden of the limb either totally, or partially. This also requires the development of new motor habits, which is difficult for the patients. However, fracture treatment by osteosynthesis has indications and the operational risk is minimal. After all, the complex and prolonged rehabilitation process is an indivisible part of the therapeutic procedure. In addition, the high risk of reoperations reaching up to $36 \%$ due to bone adhesion compromise should not be neglected.

The primary hip joint aloplastics after femoral neck fracture, although with higher operational risk, is indicated as a relatively simple and safe procedure. Moreover, there are available and reliable implants, a clear rehabilitation regimen, and a relatively low risk of complications, mainly endoprosthetic luxation (in $10 \%-24 \%$ of the cases).

The aim of this study was to build-up optimal indications for primary endoprosthesis with hemiprosthesis in femoral neck fractures and to justify the choice of an optimal hip hemiprosthetic implant (2).

The following tasks have been set: i) to optimize the indications for primary hip replacement after femoral neck fracture; ii) to carry out a oneyear comparative study of the postoperative results achieved in the application of different hemiprosthesis designs in order to choose an optimal hemiprosthetic implant, and iii) to propose recommendations for optimal therapeutic protocol for femoral neck fracture.

\section{MATERIALS AND METHODS}

The study was based on a sample of 209 hospital patients with femoral neck fracture at an average age of 85 years (range, 75 to 93 years). The patients were operated on in the Clinic of Orthopedics and Traumatology, St. Anna University Hospital of Varna, within a five-year period (between 2011 and 2015). For all the patients, a primary hip hemiarthroplasty was carried out. All the 209 patients were monitored. However, there was a drop out of 42 patients during the first three months for not attending the followup examinations. From the 209-patient cohort, 167 were followed successfully during the subsequent 12-month postoperative period.

The basic criteria for hemiarthroplasty following femoral neck fracture were grouped in two cohorts.

1. Fracture-dependent:

$\diamond$ Classification type of fracture (Garden III-IV or $\mathrm{AO}$ B2);

$\diamond$ Time duration from the fracture event (more than five days).

2. Patient-dependent:

$\diamond$ Age (over 75 years of age);

$\diamond$ Presence of neurological motor deficit;

$\diamond$ Polymorbidity (ASA III-IV).

In hemiprosthetic aloplasty, modular endoprosthesis with cement attachment of the femoral 
component and three types of the hemiprosthetic head were used (Table 1). groups with different hemiprosthetic head designs were registered (Table 2).

Table 1. Gender distribution of cases with biarticular, monoarticular elliptical and monoarticular spherical endoprosthetic heads

\begin{tabular}{lc|c|c|c|c|c|}
\hline \multirow{2}{*}{ Types of endoprosthetic heads } & \multicolumn{2}{c}{ males } & \multicolumn{2}{c}{ females } & \multicolumn{2}{c}{ total } \\
& $\mathrm{n}$ & $\%$ & $\mathrm{n}$ & $\%$ & $\mathrm{n}$ & $\%$ \\
spherical biarticular & 28 & 41.18 & 40 & 58.82 & 68 & 40.72 \\
spherical monoarticular & 21 & 41.18 & 30 & 58.82 & 51 & 30.54 \\
elliptical monoarticular & 18 & 37.50 & 30 & 62.50 & 48 & 28.74 \\
total & 67 & 40.12 & 100 & 59.88 & 167 & 100.00 \\
\hline
\end{tabular}

\section{RESULTS}

The one-year follow-up of the therapeutic results was an opportunity to detect the dynamic changes in hip functioning after a single-pole endoprosthesis (3). As a result, additional comparative analysis of the therapeutic outcomes was carried out among the different types of hemiprosthetic head designs (4). This gave space to optimize the therapeutic protocol for hip arthroplasty after femoral neck fracture.

During this follow-up, the functioning was evaluated according to the Modified Harris Hip Score (MHHS) as well as by the Visual Analogue Scale (VAS). The evaluations were carried out during the regular examinations according to the following schedule:

$\diamond$ First examination on the $6^{\text {th }}$ postoperative week;

$\diamond$ Second examination on the $12^{\text {th }}$ postoperative week;

$\diamond$ Third examination on the $26^{\text {th }}$ postoperative week, dnd

$\diamond$ Fourth examination on the $52^{\text {nd }}$ postoperative week.

We analyzed the results of the follow-up of these 167 patients with hemiarthroplasty. Up to the end of the first year after surgery, a progressive elevation in the MHHS point system as well as a regression to the disappearance of spontaneous and provoked pain regardless of the endoprosthetic design was recorded (5). This fact was an evidence of the progressive improvement of patient's status after hip hemiarthroplasty. However, at the end of the first year, some differences in the MHHS score among the patient
Table 2. Maximum and minimum values according to MHHS

\begin{tabular}{lcc} 
Prosthetic heads & $\begin{array}{c}\text { Minimal } \\
\text { values }\end{array}$ & $\begin{array}{c}\text { Maximal } \\
\text { values }\end{array}$ \\
\hline spherical biarticular & 77 & 83 \\
spherical monoarticular & 74 & 81 \\
elliptical monoarticular & 78 & 85 \\
\hline
\end{tabular}

The VAS scores showed a natural decrease in pain sensation with the passing of time from the surgical intervention despite the increasing motor activity (Table 3). The patients considered this a clear indication of improved condition. The subjective assessment of pain coincided completely with the clinical scale values (Table 3 ).

Table 3. Maximum and minimum values according to VAS

\begin{tabular}{|c|c|c|}
\hline \multirow[t]{2}{*}{ Prosthetic heads } & $\begin{array}{l}\text { Minimum } \\
\text { values }\end{array}$ & $\begin{array}{l}\text { Maximum } \\
\text { values }\end{array}$ \\
\hline & $12^{\text {th }}$ month & $12^{\text {th }}$ month \\
\hline $\begin{array}{l}\text { spherical } \\
\text { biarticular }\end{array}$ & 40 & 8 \\
\hline $\begin{array}{l}\text { spherical } \\
\text { monoarticular }\end{array}$ & 38 & 23 \\
\hline $\begin{array}{l}\text { elliptical } \\
\text { monoarticular }\end{array}$ & 32 & 25 \\
\hline
\end{tabular}

\section{DISCUSSION}

There are minimal and statistically insignificant differences when comparing the therapeutic results of these three types of prosthetic head use. The progressive increase of the values from 
the clinical assessment scales demonstrates the improvement of patient's status during the first year after surgery. The good results achieved with singlepole hip joint endoprosthesis after hip fracture for this period are of a constant magnitude. These results could be explained with the minimally invasive surgical technique applied to hip locomotor structures allowing for rapid movement recovery within an adequate rehabilitation protocol. The analysis of our therapeutic results justifies the indication of hemiarthroplasty after femoral neck fractures and the possibility of immediate postoperative limb loading, restoration of the limb support function, as well as rapid restoration of the locomotive action (6).

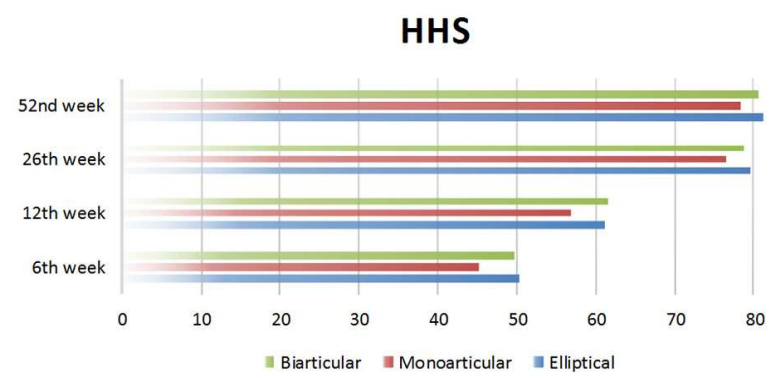

Fig. 1. Clinical assessment of the functional results according to $M H H S$

\section{VAS}

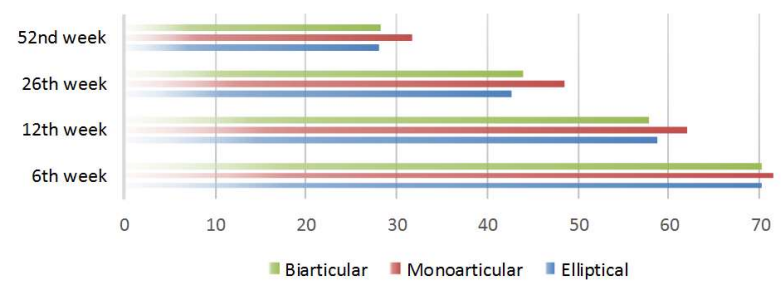

Fig. 2. VAS - subjective assessment
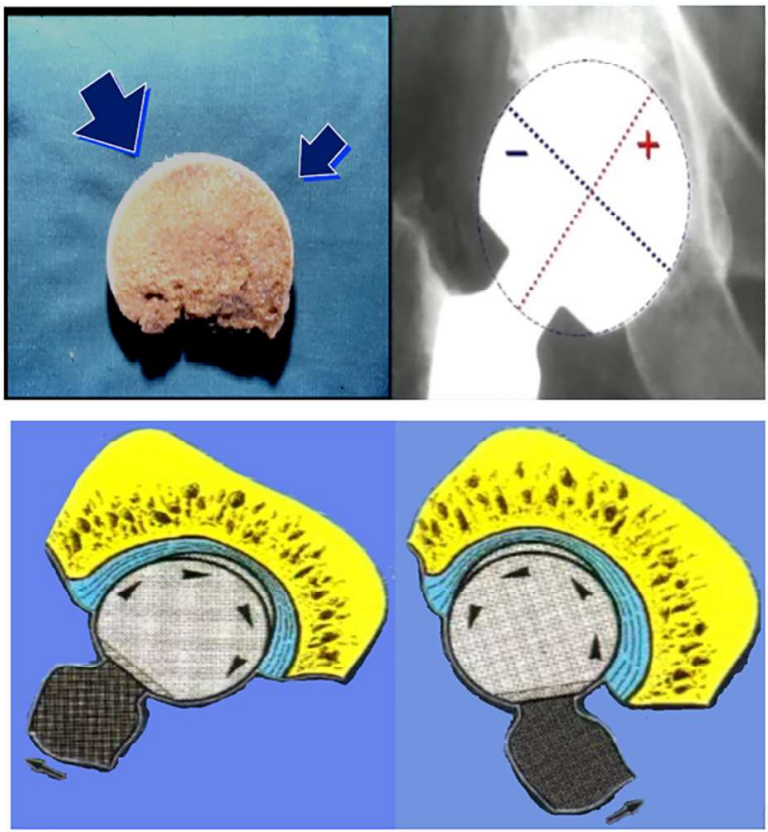

Fig. 3. Anatomical sample - cut of the femoral head and biomechanical functional hip joint scheme

The normal anatomical femoral head form is rather elliptical at the expense of a smaller diameter of its 'equator' (Fig. 3) (7).

Spherical monoarticular endoprosthetic heads provoke constant static loading of acetabular cartilage beyond its ability to reshape and to distribute symmetrically and axially the functional load on the acetabulum. Inevitably, as a result of this biomechanical deficiency, a trophic disbalance of the acetabular cartilage occurs, with expected subsequent rapid and progressive chondrolysis (Table 4) (8).

In the group with hemialoplasty by means of a monoarticular spherical endoprosthetic head, only one case of joint cartilage wearing with subsequent acetabular protrusion as the most common complication was registered. This led to conversion to total

Table 4. Registered postoperative complications with different hemiprosthetic head types

\begin{tabular}{lcccc} 
Complication & $\begin{array}{c}\text { spherical } \\
\text { monoarticular }\end{array}$ & $\begin{array}{c}\text { spherical } \\
\text { biarticular }\end{array}$ & $\begin{array}{c}\text { elliptical } \\
\text { monoarticular }\end{array}$ & Total \\
Acetabular protrusion & 1 & 0 & 0 & 1 \\
Iliac protrusion & 0 & 0 & 0 & 0 \\
Luxation & 1 & 1 & 0 & 2 \\
Aseptic loosening & 0 & 1 & 0 & 1 \\
Periprosthetic fracture & 0 & 0 & 0 & 0 \\
Perioperative mortality & 1 & 2 & 0 & 2 \\
\hline \hline
\end{tabular}


endoprosthesis. During the one-year follow-up period, with the spherical head, a luxation in the first month was recorded. It was corrected internally under X-ray control (Fig. 4) (9).

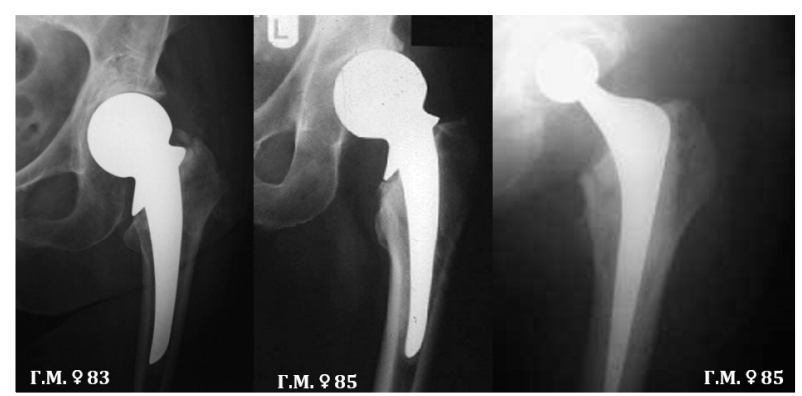

Fig. 4. Conversion to total endoprosthesis due to acetabular chondrolysis

In one patient with a spherical biarticular endoprosthetic head presenting with an unreliable social behaviour, a prosthesis luxation was diagnosed. In this case, there was a diagnostic delay by more than a month after discharge from hospital as the patient did not show up for the scheduled examinations. An open reposition was carried out and a defect of the upper acetabular edge and areas of cartilage destruction by facies lunata were found out. These findings required conversion to total hip joint endoprosthesis (Table 3).

The biarticular spherical endoprosthetic head is designed with the ambition to solve the problem of acetabular chondrolysis by 'transferrin' some movements in the polyethylene-metal articulation. However, the outer spherical contour of the endoprosthetic head is unable to compensate for the deficiency of acetabular cartilage transformation, thus causing an improper axial pressure distribution in the joint and a hygienic cartilage deficiency resulting in this endoprosthesis as well in progressive acetabular chondrolysis. In the clinical cases of this follow-up study, there was no objective radiographically detected complication of this kind up to 12 months postoperatively.

Probably, the biarticular spherical endoprosthetic head compared to the monoarticular spherical one, slows acetabular chondrolysis, a fact which can be proved objectively. In some patients, however, there is deterioration of the indicators related to hip pain and function. This does not always corre- late with the absence of $\mathrm{x}$-ray changes in the acetabular rim, e.g. joint space narrowing.

Besides the constructed elliptically shaped hemispherical hip head could be called a biological analogue. Logically, in this form of the implant, it is possible to better reproduce the elastic transformation of the hyaline acetabular cartilage and the hydraulics of the cartilage trophics, which in turn provides a symmetrical axial load in the movement and preservation of the joint lubrication.

In our cases, after hip joint hemialoplasty with a monoarticular elliptic endoprosthetic head, there is neither radiologically detected acetabular chondrolysis, nor a progressive deterioration of the subjective and functional outcomes for the follow-up period (Fig. 5) (10). Moreover, the patients from this group report the highest subjective and functional scores regarding fracture treatment.

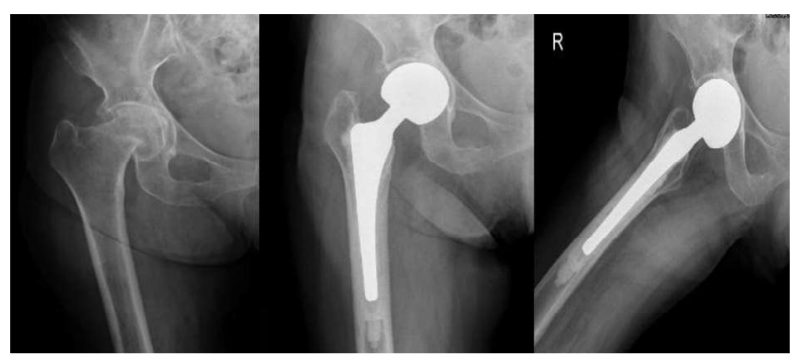

Fig. 5. Preserved joint width 12 months after elliptical hemiprosthetic head implantation

\section{CONCLUSIONS}

Based on our results, the following conclusions can be drawn:

1. The primary hip joint hemialoplasty is indicated for femoral neck fractures, Garden III or $\mathrm{IV}$, in polymorbid patients over 75 years of age. For patients in whom surgery is delayed five or more days after fracture, the primary hemialoplasty is the only rational therapeutic option.

2. The choice of the endoprosthetic implant is an essential factor in achieving predictably good therapeutic outcomes.

3. Hemiprosthesis design should warrant a minimal risk for an acetabular protrusion. The elliptical monoarticular endoprosthetic head shows better therapeutic results based on subjective, 
radiographic and functional criteria during the 12-month follow-up study.

4. Our results support the need for clear guidelines for hip joint hemiprosthesis with elliptical monoarticular head to be an implant of choice in such femoral neck fracture challenges.

\section{REFERENCES}

5. Kennedy WR. Treating femoral neck fracture with the Cathcart prosthesis: a preliminary report. Contemp Orthop. 1982;4:1-6.

6. Patel KC, Moradiya N, Gewatre P, Dasai TV. Early outcome of hemireplacement arthroplasty using cemented bipolar prosthesis in fracture neck femur in elderly: A study of 50 cases. Int J Orthop Sci. 2017;3(1):303-7. doi: 10.22271/ortho.2017.v3.ile.48

7. Andersson G, Muller Neilsen J. Results after arthroplasty of the hip with Moor's prosthesis. Acta Orthop Scand. 1972;43(5):397-410. doi: 10.3109/17453677208998960.

8. Cathcart RF. The shape of the femoral head and preliminary results of clinical use of non-spherical hip prosthesis. J Bone Joint Surg. 1972;53A:397.

9. Simone C, Patella V, Moretti B, Molfetta L. Shortterm clinical results with an elliptical femoral prosthesis. Hip Int. 1995;5(1):15-9.

10. Kenzora JE, Magaziner J, Hudson J, Hebel JR, Young Y, Hawkes W, et al. Outcome after hemiarthroplasty for femoral neck fractures in the elderly. Clin Orthop Relat Res. 1998; (348):51-8.

11. Angelini S, Biancalani D, Martelli F, Davini PG, De Luca L. The Cephalic Prosthesis "ELLITICA" for Femoral Neck Fractures. Regione Toscana - Local Health Authority USL 11 U.O.S. of Traumatology Ospedale San Giuseppe

12. Bullough PG, Goodfellow JW, O'Conner JJ. The relationship between degeneration changes and load-bearing in the human hip. J. Bone Joint Surg. 1973;55(4):746-58.

13. Coleman SH, Bansal M, Cornell CN, Sculco TP. Failure of bipolar hemiarthroplasty: a retrospective review of 31 consecutive bipolar prostheses converted to total hip arthroplasty. Am J Orthop (Belle Mead NJ). 2001;30(4):313-9.

14. Pipino E, Molfetta L. The 'Cathcart orthocentric' elliptical head endoprosthesis. A long-term clinical and radiographic study. Ital J Orthop Traumatol. 1989;15(1):5-14. 